\title{
Performance Evaluation of Compound Barker Codes using Cascaded Mismatched Filter Technique
}

\author{
B. Kiranmai \\ Gitam Institute of Technology \\ Department of ECE, \\ Visakhapatnam, India
}

\author{
P. Rajesh Kumar \\ Andhra University \\ Department of ECE, \\ Visakhapatnam, India
}

\begin{abstract}
The major advantages of pulse compression are low pulsepower which makes it suitable for solid-state devices, higher maximum range, good range resolution and better jamming immunity. The matched filter is the optimal linear filter for maximizing the signal to noise ratio (SNR) in the presence of additive stochastic noise. Pulse compression is an example of matched filtering. But this matched filter output consists of unwanted but unavoidable side lobes. For multiple-target radar, the side lobes of the compressed pulse must be considered in the system design because of the likelihood of false alarms. At the receiver the signal processor uses weighting filters which are not matched to the transmitted waveform. When this filter is not matched to the transmitted waveform then filter output consists of unwanted but unavoidable side lobes. In this paper a new technique is proposed to suppress the side lobes of radar signals that result from standard matched filtering. This technique produces better peak side lobe ratio than all other conventional side lobe reduction techniques. In simulation the results of this filter technique for compound Barker codes is compared with the other side lobe reduction techniques.
\end{abstract}

\section{Keywords}

Barker code, Compound Barker code, Peak side lobe Ratio, Amplitude shift code, Cascaded filter

\section{INTRODUCTION}

Pulse compression originated with the desire to amplify the transmitted impulse (peak) power by temporal compression. It is a method which combines the high energy of a long pulse width with the high resolution of a short pulse width. Since each part of the pulse has unique frequency, the returns can be completely separated. This modulation or coding can be either FM (frequency modulation) or linear (chirp radar) or nonlinear, time-frequency-coded waveform (e.g. Costas code) or PM (phase modulation). The receiver is able to separate targets with overlapping of noise. The received echo is processed in the receiver by the matched filter. The matched filter readjusts the relative phases of the frequency components so that a narrow or compressed pulse is again produced. The radar therefore obtains a better maximum range than it is expected because of the conventional radar equation. The ability of the receiver to improve the range resolution over that of the conventional system is called the pulse compression ratio (PCR) $[1,5]$. When a target echo signal is passed through a matched filter and outputs a spikelike main lobe and some unwanted but unavoidable noise-like side lobes. Side lobes are undesirable because noise and jammers located in the side lobes may interfere with the target proceeds in the main lobe. These side lobes can form spurious targets or mask the main lobe of weak target echo signals at adjacent range cells [5]. To prevent these problems, a binary code has to be designed whose auto correlation function main lobe-to-peak-side lobe ratio is maximized for a given code length. Enormous efforts have been dedicated to design a good auto correlation function property for binary sequences $[2,3,4]$. Furthermore, we have to employ a side lobe reduction filter to achieve an adequate main lobe-to-peak-side lobe ratio. The first method is by using a matched filter to perform the pulse compression correlation and this output of the matched filter is cascaded with the mismatched filter to suppress the side lobes. In this contest the technique was used to suppress the side lobes is the amplitude shift code technique [6],[7],[8]. In this technique the output of the matched filter consists of the peak in positive phase and the side lobes are in negative phase. Amplitude shifting technique shifts the positive phase up above zero and the negative in phase shifts down below zero. So By using a simple circuit the positive peak can be restored. In this way better side lobe suppression achieved with simple circuit and with lesser hard ware complexity. And the other technique is used to suppress the side lobes by using cascaded mismatched filter proposed by Indranil Sarkar and Adly T. Fam [9].In this technique they proposed compound barker codes instead of Barker codes. Because the length of the Barker codes are limited to the 13 . Because of their uniform sidelobe level property, the length of the Barker code should be increased. So in this paper compound Barker codes with Gaussian noise added are used and simulated the results for all these techniques .

\section{BARKER CODED WAVEFORM}

The binary code consists of a sequence of either +1 and -1 . The phase of the transmitted signal alternates between 0 and $180^{\circ}$ in accordance with the sequence of elements, in the phase code, as shown on the figure1. Since the transmitted frequency is usually not a multiple of the reciprocal of the sub pulse width, the coded signal is generally discontinuous at the phase-reversal points. The selection of the so called random 0 , $\pi$ phases is in fact critical. The binary choice of 0 or $\pi$ phase for each sub-pulse may be made at random. However, some random selections may be better suited than others for radar application. One criterion for the selection of a good "random" phase-coded waveform is that its autocorrelation function should have equal time side-lobes. The binary phasecoded sequence of $0, \pi$ values that result in equal side-lobes after passes through the matched filter is called a Barker code.

\section{COMPOUND BARKER CODE}

Compound Barker Codes is demonstrated using pair wise combinations of Barker Codes of length 13, 11, 7and 5. If a code $\mathrm{C}_{\mathrm{N} 1}$ of length $\mathrm{N}_{1}$ is compounded with another code $\mathrm{C}_{\mathrm{N} 2}$ of length $\mathrm{N}_{2}$, the $\mathrm{z}$-domain representation for such compounding is given by $\quad \mathrm{C}_{\mathrm{N} 1, \mathrm{~N} 2}(\mathrm{z})=\mathrm{C}_{\mathrm{N} 2}(\mathrm{z}) \quad \mathrm{X} \mathrm{C}_{\mathrm{N} 1}(\mathrm{z})$ Where $\mathrm{C}_{\mathrm{N} 1}(\mathrm{z})$ is the outer code and $\mathrm{C}_{\mathrm{N} 2}(\mathrm{z})$ is the inner code. In 
the time domain, the inner code is repeated a number of times equal to the number of bits in the outer code. In each repetition, the inner code is phase inverted or not depending on whether the corresponding bit in the outer is -1 or +1 respectively. Let us consider the Barker Codes of length 7 and 5 as $\quad \mathrm{B}_{7}=\left\{\begin{array}{lllllll}1 & 1 & 1 & -1 & -1 & 1 & -1\end{array}\right\} \quad \mathrm{B}_{5}=\left\{\begin{array}{lllll}1 & 1 & 1 & -1 & 1\end{array}\right\}$

Either of these codes could be compounded with the other to produce a code of length 35 . If the outer code is length 5 and the inner code is length 7 , the compound code is denoted by $\mathrm{B}_{5} \quad \mathrm{~B}_{7}$, where represents the Kronecker product.

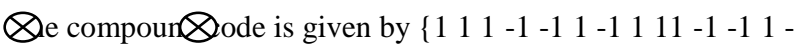
1111 -1 -1 1 - -1 - -1 - 1 -1 11 -1 1111 - 1 - -1 1 $1-1$ \}

\section{AMPLITUDE SHIFT CODE PULSE COMPRESSION METHOD}

A new pulse compression method is used to generate a flat uniform side lobe pattern similar to those obtainable from Compound Barker codes with less complexity in the structure and to reduce the side lobe level. Because the main peak is always positive phase and the side lobes are at negative phase. An amplitude shift is added to transmitted wave along with phase modulation. Amplitude shifting is the wave multiplied with the positive phase i.e +1 then it shifted up above zero. And the negative phase is multiplied by -1 then it shifted by below zero. Because of this the peak output amplitude will be shifted above zero and while the side lobes which are in negative phase and negative in amplitude will be shifted down below zero. Because of this the positive peak will be in one side and the negative peak will be in the below zero side. So any electronic circuit can be used to detect the peak signal .The amplitude of the side lobes can be discarded .

The block diagram for amplitude shifted code pulse compression technique is as shown in figure (1)

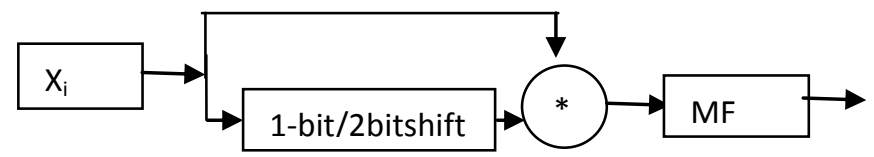

Fig.1

Where $\mathrm{Xi}$ is Compound Barker code, MF is Matched filter.

In figure 1 Compound Barker code signal is combined with one-bit/two bit version of itself and the resulting signal is auto correlated output will be having reduction in side lobes.

\section{CASCADING MISMATCHED FILTER TECHNIQUE}

To analyze the cascaded filters, consider the filter arrangement as shown in figure 2 always less than $\mathrm{N}$. The third filter is characterized by the number $\mathrm{O}$ of pulses processed and by the weight vector $\mathrm{C}$.

Let us consider the incoming Barker code be $X(z)$. A white noise has been added to this incoming signal. This Barker code with noise is input to the matched filter .Then the output of the matched filter is input to the mismatched filter and that should be designed to suppress the side lobes at the matched filter. Even though matched filter is the optimal filter to maximize the SNR when an uncorrelated Gaussian noise is present. By designing inverse transfer function of the autocorrelation function of the matched filter then the side lobes can be minimized. So the autocorrelation function of matched filter is given as

$\mathrm{R}(\mathrm{z})=\mathrm{X}(\mathrm{z})^{*} \mathrm{x}\left(\mathrm{z}^{-1}\right)$ this can be written as $\mathrm{R}(\mathrm{z})=\mathrm{N}+\sum_{n=1}^{\mathrm{N}-1} \operatorname{Sn}\left(z^{n}+z^{-n}\right) \ldots \ldots \ldots .(1)$

In equation $1, \mathrm{~N}$ is the magnitude of the main lobe and the second term represents side lobes. So to suppress the side lobes the transfer function of mismatched filter is inverse of this.

The transfer function of the required mismatched filter for the compound code is of the form

$\mathrm{M}_{\mathrm{N} 1, \mathrm{~N} 2}(\mathrm{z})=\left[\mathrm{B}_{\mathrm{N} 2}\left(\mathrm{z}^{-1}\right) \cdot \mathrm{F}_{\mathrm{N} 2}(\mathrm{z})\right] \cdot\left[\mathrm{B}_{\mathrm{N} 1}\left(\mathrm{z}^{-1}\right) \cdot \mathrm{F}_{\mathrm{N} 1}(\mathrm{z})\right]$

equation 4 can be rewrite by grouping the matched filter terms and mismatched filter terms, then

$$
\mathrm{M}_{\mathrm{N}}(\mathrm{z})=\left[\mathrm{B}_{\mathrm{N} 2}\left(\mathrm{z}^{-1}\right) \cdot\left[\mathrm{B}_{\mathrm{N} 1}\left(\mathrm{z}^{-1}\right)\right] \cdot\left[\mathrm{F}_{\mathrm{N} 1}(\mathrm{z}) \cdot \mathrm{F}_{\mathrm{N} 2}(\mathrm{z})\right]\right. \text {. }
$$

\section{PERFORMANCE EVALUATION CALCULATIONS FOR COMPOUND BARKER CODE}

Peak Side Lobe Ratio (PSLR) is equal to the ratio of the energy in the chirp corresponding to the highest side lobe normalized by the energy in the chirp at the peak response.

PSLR $=\mid$ Yn peak sidelobe $\left.\right|^{2} /|Y 0|^{2}$

Where $\mathrm{Y}_{0}=$ peak voltage response of the filter

$$
\mathrm{Yn}=\text { voltage of } \mathrm{n}^{\text {th }} \text { range side lobe }
$$

\section{SIMULATION RESULTS}

Therefore $\mathrm{R}(\mathrm{z})=\mathrm{N}\left(1+1 / \mathrm{N} \sum_{n=1}^{\mathrm{N}-1} \mathrm{Sn}\left(z^{n}+z^{-n}\right)\right)$ the transfer function of mismatched filter is

$$
\mathrm{h}(\mathrm{z})=\frac{\mathrm{N}}{\mathrm{R}(\mathrm{z})}=\frac{1}{1+1 / \mathrm{N} \sum_{n=1}^{\mathrm{N}-1} \operatorname{Sn}\left(z^{n}+z^{-n}\right)}
$$

After rationalizing the transfer function for the number of stages required and optimizing the transfer function for the better side lobe reduction, the final equation will be

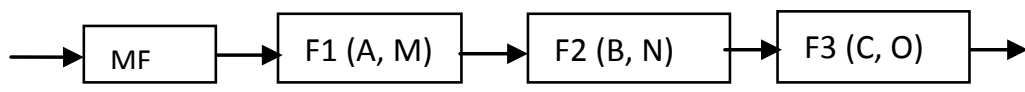

Fig.2

Figure 2 shows three stages cascading mismatched filter. This mismatched filter is aims to suppress the side lobes that produced at the receiver. The first filter is characterized by the number $\mathrm{M}$ of pulses processed and by the weight vector $\mathrm{A}$. The second filter is characterized by the number $\mathrm{N}$ of pulses processed and by the weight vector $\mathrm{B}$. The number $\mathrm{M}$ is

$$
\begin{aligned}
\mathrm{F}(\mathrm{z})= & {\left[1-1 / \mathrm{N} \sum_{n=1}^{\mathrm{N}-1} \operatorname{Sn}\left(z^{n}+z^{-n}\right)\right] } \\
& {\left[1+1 / \mathrm{N}^{2}\left\{\sum_{n=1}^{\mathrm{N}-1} \operatorname{Sn}\left(z^{n}+z^{-n}\right)\right\}^{2}\right] } \\
& {\left[1+1 / \mathrm{N}^{4}\left\{\sum_{n=1}^{\mathrm{N}-1} \operatorname{Sn}\left(z^{n}+z^{-n}\right)\right\}^{4} .\right.}
\end{aligned}
$$


Equation 2 is the optimized transfer function for the three stages cascaded mismatched filter .

For a compound Barker codes if $\mathrm{B}_{\mathrm{N} 1}$ is compounded with $\mathrm{B}_{\mathrm{N} 2}$ then the autocorrelation function is denoted as $R_{N}(z)$ is given by $\mathrm{R}_{\mathrm{N} 1, \mathrm{~N} 2}(\mathrm{z})=\mathrm{R}_{\mathrm{N} 2}(\mathrm{z}) \cdot \mathrm{R}_{\mathrm{N} 1}\left(\mathrm{z}^{\mathrm{N} 2}\right)$

The mismatched filter for compound Barker codes can be implemented by cascading two filters $\mathrm{M}_{\mathrm{N} 1}(\mathrm{z})$ and $\mathrm{M}_{\mathrm{N} 2}(\mathrm{z})$. So the individual mismatched filters are given by

$$
\mathrm{M}_{\mathrm{N} 1}(\mathrm{z})=\mathrm{B}_{\mathrm{N} 1}\left(\mathrm{z}^{-1}\right) \cdot \mathrm{F}_{\mathrm{N}}(\mathrm{z})
$$

In equation $3 \mathrm{~F}_{\mathrm{N}}(\mathrm{z})$ is the transfer function of three stages cascaded mismatched filter. Similarly for

$\mathrm{M}_{\mathrm{N} 2}(\mathrm{z})=\mathrm{B}_{\mathrm{N} 2}\left(\mathrm{z}^{-1}\right) \cdot \mathrm{F}_{\mathrm{N}}(\mathrm{z})$ where $\mathrm{B}_{\mathrm{N} 1}$ and $\mathrm{B}_{\mathrm{N} 2}$ are the Barker codes.

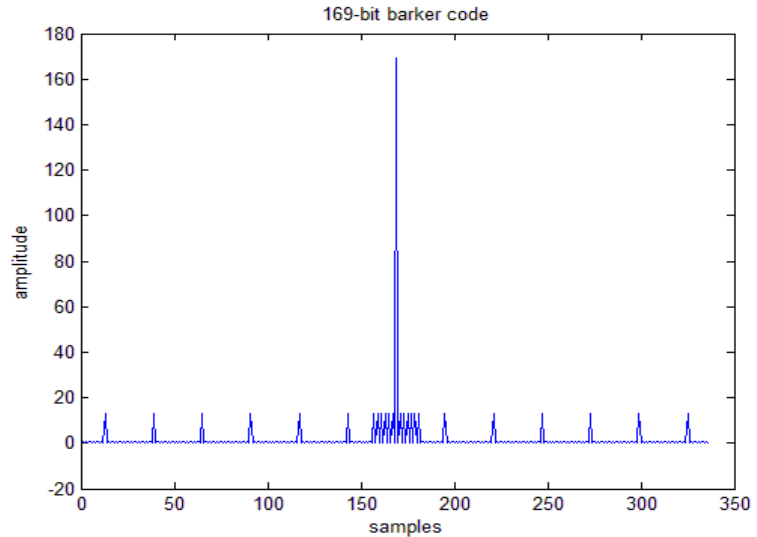

Fig3.ACF of Compound Barker code 169

In figure3 shows the autocorrelation function of the Compound Barker code of length 169(13x13).

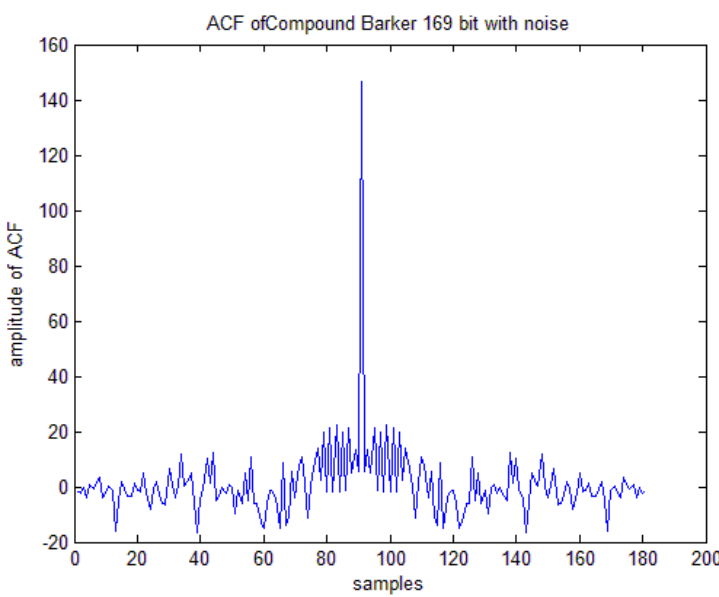

Fig 4 ACF of compound Barker code with noise

In figure 4 the autocorrelation function of Compound Barker code of length 169 with Gaussian noise is added.

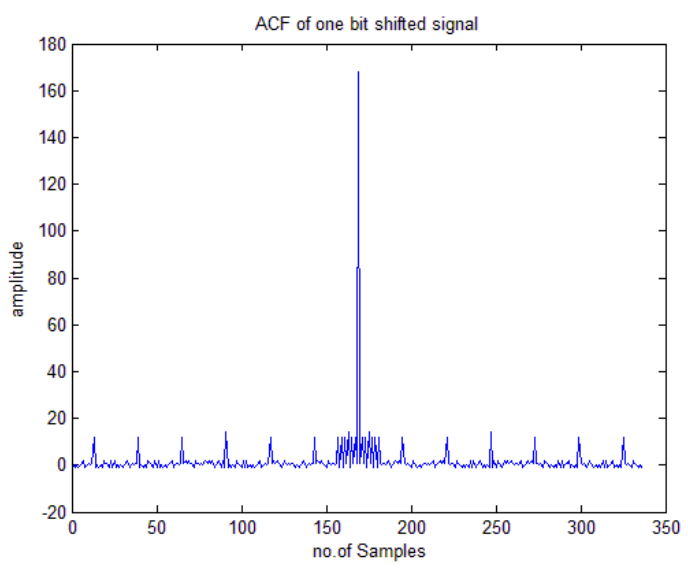

Figure $5 \mathrm{ACF}$ of combined code of length 169

In figure 5 shows Compound Barker code of length 169 is added with the 1-bit shifted Compound Barker code to get the amplitude difference directly for the Compound Barker code of length 169 .

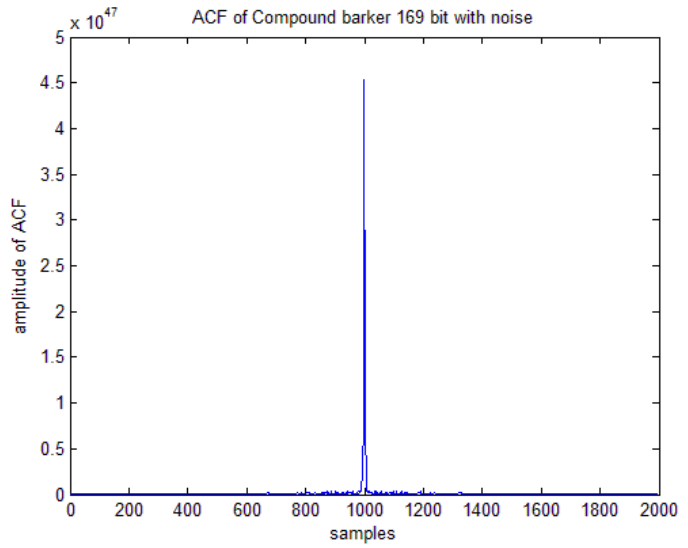

Figure. 6 ACF of compound Barker code of length 169

In figure 6 shows three stages cascading mismatched filter output of Compound Barker code of length 169.

Table 1

\begin{tabular}{|ll|l|l|l|l|}
\hline $\begin{array}{l}\text { Compound } \\
\text { Barker code } \\
\text { Inner outer }\end{array}$ & $\begin{array}{l}\text { Code } \\
\text { Length }\end{array}$ & $\begin{array}{l}\text { 1-bit } \\
\text { shift } \\
\text { code } \\
\text { PSLR }\end{array}$ & $\begin{array}{l}\text { 2bit } \\
\text { shift } \\
\text { code } \\
\text { PSLR }\end{array}$ & $\begin{array}{l}\text { Cascaded } \\
\text { filter } \\
\text { PSLR }\end{array}$ \\
\hline 5 & 5 & 25 & 63.96 & 63.97 & 98.1 \\
\hline 7 & 5 & 35 & 74.3 & 74.4 & 93.6 \\
\hline 11 & 5 & 55 & 79.8 & 79.9 & 95.8 \\
\hline 13 & 5 & 65 & 51.14 & 51.14 & 90.02 \\
\hline 5 & 7 & 35 & 34.98 & 43.09 & 91.07 \\
\hline 5 & 11 & 55 & 47.96 & 47.7 & 88.02 \\
\hline 5 & 13 & 65 & 47.49 & 47.49 & 90.02 \\
\hline 13 & 13 & 169 & 88.67 & 88.5 & 106.01 \\
\hline
\end{tabular}


Table 1 shows different Compound Barker codes generated by using Barker code lengths of 5,7,11 and 13.The PSLR values are calculated for Amplitude shifted code and cascaded mismatched filter. These PSLR values are compared. By examining the results the three stage cascading mismatched filter obtains better side lobe level reduction ratio than other technique. But Amplitude shift code method is having better side lobe reduction with lesser hard ware complexity.

\section{CONCLUSIONS}

The other mismatched filters are observed to be suppresses the side lobes more than that of windowing technique. These filters are applicable to any binary coding signals. But.The hard ware structure is very complicated for higher stages of mismatched filters. Amplitude shift code method achieves grater side lobe reductions with the simple circuitry and with less number of adders and multipliers. But the cascading mismatched filtering method achieves greater side lobe levels than the Amplitude side lobe reduction technique , But the hard ware structure complicated for higher stages than the Amplitude shift code method.

\section{REFERENCES}

[1] Skolnik, M. I. Introduction to Radar Systems (3rd ed.). New York: McGraw-Hill, 2001, 339-369.

[2] Rihaczek, A. W. and Golden, R. M. Range side lobe suppression for Barker codes. IEEE Transactions on Aerospace and Electronic Systems, AES-7 (Nov. 1971), 1087-1092.
[3] Baden, J. M. and Cohen, M. N. Optimal side lobe suppression for bi phase codes In Proceedings of the IEEE Tele systems Conference, 1991, 127-131.

[4] Chen Xiao Hua and Juhani Oskman, A New Algorithm to Optimize Barker Code Side lobe and Suppression Filter, IEEE Transactions on Aerospace and Electronic Systems, Vol AES-26, No. 4, July 1990, pp 673-677.

[5] Nadav Levanon, Eli Mozeson, "Radar Signals", John Wiley \& Sons, 2004, Hoboken, New Jersey

[6] Talal Darwich, Charles Cavanaugh Amplitude Shifting for Sidelobes Cancellation pulse compression Technical Report TR-2006-4

[7] A New Pulse Compression Technique for Poly phase Codes in Radar Signals International Symposium on Devices MEMS, Intelligent Systems \& Communication (ISDMISC) 2011 Proceedings published by International Journal of Computer Applications® (IJCA)

[8] Performance Evaluation of Barker Codes using New Pulse Compression Technique International Journal of Computer Applications (0975 - 8887) Volume 107 - No. 20, December 2014

[9] Indranil Sarkar and Adly T. Fam ,Factored Multiplicative Mismatched Filters for Compound Barker Codes in the publications of the IEEE 1-4244-0284-0/07 